\title{
Disturbance decoupling using a novel approach to integral sliding-mode
}

\author{
Tobias Posielek ${ }^{\dagger}$, Kai Wulff ${ }^{\ddagger * *}$ and Johann Reger ${ }^{\ddagger}$
}

\begin{abstract}
We propose a novel approach to design an integral sliding mode control (ISMC) for a nonlinear system in regular form. The control method is capable to compensate a class of matched and, in particular, unmatched uncertainties with respect to some given output. Conditions for stability and decoupling are presented. For the case of linear nominal dynamics these conditions take a very simple form. A direct comparison to conventional design methods for ISMC is given and the concepts are illustrated using a simulation example.
\end{abstract}

\section{INTRODUCTION}

Sliding mode control is a control technique, widely known for its robustness properties. Disturbances that fulfil a matching condition are completely repelled once the system is in sliding mode. Integral sliding mode (ISM) is a special kind of sliding mode concept that has been introduced by Utkin in [1]. The main feature of this concept is the introduction of an additional integrator state that allows to initialise the system on the sliding manifold and thus eliminate the reaching phase. This establishes the robustness to matched uncertainties throughout the entire system response. This property makes an integral sliding mode controller (ISMC) a common choice for systems with matched external disturbances or model uncertainties.

In [2] an adaptive ISMC is proposed to control the longitudinal rotation of a tilt-rotor aircraft. The ISMC guarantees robustness to bounded matched uncertainties such as sensor noise. An ISMC for a two wheeled-mobile robot is given in [3] to completely annihilate the influence of the joint friction acting on the system.

As the sliding motion is completely insensitive to matched uncertainties only, but not to unmatched ones, minimising the effects of the latter is part of designing an ISMC. One way to deal with unmatched uncertainties in sliding mode control is to find a transformation which results in an integrator chain system which does not contain unmatched uncertainties. However, this requires an observer to estimate the uncertainties [4], [5]. In [6] the integral sliding mode is studied incorporating the effect of the unmatched uncertainties on the reduced dynamics. In [7] a projection matrix is proposed that minimises the effect of the matched disturbance in the reduced dynamics. These results are extended in [8]. In [9] an integral HOSM technique is proposed that utilises the socalled hierarchical quasi-continuous controller design [10]. An extension using a backstepping approach is given in [11].

\footnotetext{
*Corresponding author: kai.wulffetu-ilmenau.de

${ }^{\dagger}$ Author is with the Institute of System Dynamics and Control, German Aerospace Center (DLR), Muenchner Str. 20, D-82234 Wessling, Germany

$\ddagger$ Authors are with the Control Engineering Group, Technische Universität Ilmenau, P.O. Box 1005 65, D-98684, Ilmenau, Germany
}

Those conventional ISMC designs choose a nominal control and a projection matrix. This defines the dynamics of the integral state as well as the sliding surface which yields the desired nominal (reduced) dynamics.

The method that we propose chooses the dynamics of the integral state directly in accordance with the design objective and uses the projection matrix to shape the reduced dynamics. This results in reduced dynamics that are typically of lower order than in conventional designs. Furthermore, we derive conditions for which the desired output is completely decoupled from a class of unmatched uncertainties.

Similar decoupling approaches have been investigated in [12] and [13]. Both use an input-output linearisation of the system with uncertainties acting only on the internal dynamics. Other approaches utilize an observer to estimate the uncertainty for subsequent compensation, e.g. [14], [15], [16].

The paper is organised as follows. Section II defines the system class and the control objective. In Section III we propose our design method for the ISMC and present stability and decoupling conditions. Moreover, the resulting control is directly compared to the conventional design. Section IV considers the case of linear time-invariant nominal dynamics and derives design conditions for this system class. In Section V we give a simulation example to illustrate and compare the design to conventional approaches.

\section{Problem Definition}

We consider nonlinear systems of the form

$$
\begin{aligned}
& \dot{x}_{1}=f_{1}\left(x_{1}, x_{2}\right)+\phi_{1}\left(x_{1}, x_{2}\right) \\
& \dot{x}_{2}=f_{2}\left(x_{1}, x_{2}\right)+b_{2}\left(x_{1}, x_{2}\right) u+\phi_{2}\left(t, x_{1}, x_{2}\right)
\end{aligned}
$$

where $x_{1}(t) \in \mathbb{R}^{n-1}$ and $x_{2}(t) \in \mathbb{R}$ denote the state, and the control input is $u(t) \in \mathbb{R} . f_{1}, f_{2}$ and $b_{2}$ are sufficiently smooth vector fields of matching dimensions, where $f_{i}(0,0)=0, i=1,2$. The system is subject to the uncertainties $\phi_{1}: \mathbb{R}^{n} \rightarrow \mathbb{R}^{n-1}$ and $\phi_{2}: \mathbb{R} \times \mathbb{R}^{n} \rightarrow \mathbb{R}$ satisfying some boundary conditions given in the respective sections. To ensure controllability, we require $b_{2}\left(x_{1}, x_{2}\right) \neq 0$ for all $x_{1}, x_{2}$. Note that (1) is in regular form [17]. The unmatched uncertainty is denoted as $\phi_{1}$, while $\phi_{2}$ denotes the matched uncertainty. For convenience of notation we introduce the total state $x:=\left(x_{1}^{\top}, x_{2}\right)^{\top}$ as well as $f(x):=$ $\left(f_{1}\left(x_{1}, x_{2}\right)^{\top}, f_{2}\left(x_{1}, x_{2}\right)\right)^{\top}, b(x):=\left(0, b_{2}\left(x_{1}, x_{2}\right)\right)^{\top}$, and the overall uncertainty $\phi:=\left(\phi_{1}^{\top}, \phi_{2}\right)^{\top}$.

The control objective is to guarantee the existence of an asymptotically stable equilibrium of the closed-loop system and

$$
\lim _{t \rightarrow \infty} h\left(x_{1}(t), x_{2}(t)\right)=0
$$


for some uniformly continuous output function $h$ and unknown $\phi_{1}$ and $\phi_{2}$. Furthermore, the matched disturbance $\phi_{2}$ shall be compensated on $h$ for all $t \geq 0$. Moreover, we shall derive conditions for which a part of the unmatched disturbance $\phi_{1}$ is completely compensated on $h$ for all $t \geq 0$.

\section{Proposed Integral Sliding-Mode Design}

In this section we propose an integral sliding mode controller that solves the control task. While the discontinuous part of the sliding mode control ensures robustness against the matched uncertainty, the designed integral state guarantees a vanishing stationary error of the desired output $h$, as in (2), when unmatched uncertainties are present.

Define the integrator state $z$ as per

$$
\dot{z}=h\left(x_{1}, x_{2}\right)
$$

in view of (2). We choose the switching function

$$
s\left(x_{1}, x_{2}, z\right)=g\left(x_{1}, x_{2}\right)+z
$$

where $g$ is to be selected such that $\frac{\partial g\left(x_{1}, x_{2}\right)}{\partial x_{2}} \neq 0$ for all $\left(x_{1}, x_{2}\right) \in \mathbb{R}^{n}$. Then there is a function $l: \mathbb{R}^{n} \rightarrow \mathbb{R}$ ensuring

$$
s\left(x_{1}, x_{2}, z\right)=0 \Leftrightarrow x_{2}=l\left(x_{1}, z\right) .
$$

In order to obtain a more compact notation, we define

$$
G(x):=\left[\begin{array}{ll}
G_{1}(x)^{\top} & G_{2}(x)
\end{array}\right]:=\left[\begin{array}{ll}
\frac{\partial g\left(x_{1}, x_{2}\right)}{\partial x_{1}} & \frac{\partial g\left(x_{1}, x_{2}\right)}{\partial x_{2}}
\end{array}\right]
$$

where $G_{1}(x) \in \mathbb{R}^{n-1}$ and $G_{2}(x) \in \mathbb{R}$. Then the control law that yields $\dot{s}=-\rho \operatorname{sgn}(s)$ for $\phi \equiv 0$ and $\rho>0$ is given by

$$
u(x)=\frac{-1}{G_{2}(x) b_{2}(x)}(G(x) f(x)+h(x)+\rho \operatorname{sgn}(s)) .
$$

Choosing the initial state $z(0)$ according to

$$
z(0)=-g\left(x_{1}(0), x_{2}(0)\right)
$$

guarantees sliding-mode for $t=0$. The reduced dynamics including the uncertainties then takes the shape

$$
\begin{aligned}
\dot{x}_{1} & =f_{1}\left(x_{1}, l\left(x_{1}, z\right)\right)+\phi_{1}\left(x_{1}, x_{2}\right) \\
\dot{z} & =h\left(x_{1}, l\left(x_{1}, z\right)\right) .
\end{aligned}
$$

\section{A. Stability analysis}

In this section we shall derive a sufficient condition for stability of the proposed approach. In order to formulate our result, we shall note a number of assumptions in terms of the vector $w:=\left(x_{1}^{\top}, z\right)^{\top} \in \mathbb{R}^{n}$.

For $\phi_{1}(0) \neq 0$ the origin is not an equilibrium point of the reduced dynamics (8). Thus, we require

Assumption 1 There exists an equilibrium point $w^{*}:=\left(x_{1}^{* \top}, z^{*}\right)^{\top}$ with $x_{2}^{*}=l\left(x_{1}^{*}, z^{*}\right)$ such that

$$
\begin{aligned}
f_{1}\left(x_{1}^{*}, l\left(x_{1}^{*}, z^{*}\right)\right)+\phi_{1}\left(x_{1}^{*}, l\left(x_{1}^{*}, z^{*}\right)\right) & =0, \\
h\left(x_{1}^{*}, l\left(x_{1}^{*}, z^{*}\right)\right) & =0 .
\end{aligned}
$$

We further require that (8) meets linear growth bounds:
Assumption 2 There exists a neighbourhood $W^{*}$ of the origin such that for all $w^{*} \in W^{*}$ there is a neighbourhood $W$ of $\left(x_{1}^{* \top}, z^{*}\right)^{\top}$ where for all $w \in W$ holds:

$$
\begin{gathered}
\| f_{1}\left(x_{1}, l\left(x_{1}, z\right)\right)-f_{1}\left(x_{1}^{*}, l\left(x_{1}^{*}, z^{*}\right)\right) \\
-f_{1}\left(\Delta x_{1}, l\left(\Delta x_{1}, \Delta z\right)\right)\left\|\leq \gamma_{1}\right\| \Delta w \|, \\
\left\|h\left(x_{1}, l\left(x_{1}, z\right)\right)-h\left(\Delta x_{1}, l\left(\Delta x_{1}, \Delta z\right)\right)\right\| \leq \gamma_{3}\|\Delta w\| .
\end{gathered}
$$

with $\Delta x_{1}=x_{1}-x_{1}^{*}, \Delta z=z-z^{*}$ and $\Delta w=w-w^{*}$.

Furthermore, $g$ in switching function (4) has to be chosen such that the origin of the reduced dynamics (8) is asymptotically stable in the absence of uncertainties, i.e. $\phi_{1} \equiv 0$ :

Assumption 3 There exists a Lyapunov function $V$ that for all $\Delta w$ with $w^{*} \in W^{*}$ and $w \in W$ satisfies the conditions

$$
\begin{gathered}
c_{1}\|\Delta w\|^{2} \leq V(\Delta w) \leq c_{2}\|\Delta w\|^{2}, \\
\frac{\partial V(\Delta w)}{\partial \Delta w}\left[\begin{array}{c}
f_{1}\left(\Delta x_{1}, l\left(\Delta x_{1}, \Delta z\right)\right) \\
h\left(\Delta x_{1}, l\left(\Delta x_{1}, \Delta z\right)\right)
\end{array}\right] \leq-c_{3}\|\Delta w\|^{2}, \\
\left\|\frac{\partial V(\Delta w)}{\partial \Delta w}\right\| \leq c_{4}\|\Delta w\| .
\end{gathered}
$$

Note that Assumption 3 leads to uniform asymptotic stability of the closed loop system. Relaxing this stability requirements may lead to less restrictive conditions.

Finally, we have some requirements for the overall uncertainty $\phi:=\left(\phi_{1}^{\top}, \phi_{2}\right)^{\top}$ and the unmatched part $\phi_{1}$.

Assumption 4 The uncertainty $\phi(x)$ projected by $G(x)$ is locally bounded by $\phi^{\text {sup }}>0$, i.e. there exists an invariant set $\mathbb{X} \subseteq \mathbb{R}^{n}$ such that for all $x \in \mathbb{X}$ holds:

$$
\|G(x) \phi(t, x)\| \leq \phi^{\text {sup }} .
$$

Further, the unmatched uncertainty $\phi_{1}$ shall satisfy a linear growth bound $\gamma_{2}>0$ on the sliding manifold for all $w^{*} \in$ $W^{*}$ and $w \in W$ :

$$
\left\|\phi_{1}\left(x_{1}, l\left(x_{1}, z\right)\right)-\phi_{1}\left(x_{1}^{*}, l\left(x_{1}^{*}, z^{*}\right)\right)\right\| \leq \gamma_{2}\|\Delta w\| .
$$

Note that condition (13) is often required to hold for all $x \in \mathbb{R}^{n}$. However, this may turn out too restrictive for many practical applications and can be relaxed if an invariant subset $\mathbb{X} \subseteq \mathbb{R}^{n}$ exists as stated in our assumption.

Theorem 1 Consider system (1) augmented by (3) with the integral sliding mode control law (6) subject to the Assumptions 1 to 4 . Then the sliding manifold $s\left(x_{1}, x_{2}, z\right)=0$ is reached in finite time if $\rho \geq \phi^{\text {sup }}+\varepsilon$ for $\varepsilon>0, x(0) \in \mathbb{X}$.

Proof: We apply a standard technique for showing this property and take a Lyapunov function with $\dot{V} \leq-\alpha V^{\frac{1}{2}}$, $\alpha>0$. This gives finite time convergence to $s=0$ [18]. Consider $V(s)=\frac{1}{2} s^{2}$. It follows

$$
\begin{aligned}
\dot{V}=s \dot{s}=s & (G(x) \dot{x}+h(x)) \\
=s & \left(G_{1}(x) f_{1}(x)+G_{2}(x) f_{2}(x)\right. \\
& \left.+G_{2}(x) b_{2}(x) u(x)+G(x) \phi(x)+h(x)\right) .
\end{aligned}
$$


Substituting the control law (6) we obtain

$$
\begin{aligned}
\dot{V} & =s(G(x) \phi(x)-\rho \operatorname{sgn}(s)) \leq\|G(x) \phi(x)\|\|s\|-\rho\|s\| \\
& \leq-\varepsilon\|s\| .
\end{aligned}
$$

Then we can state the main theorem of this section.

Theorem 2 Consider system (1) augmented by (3) with the integral sliding mode control law (6) and initialisation (7). Let Assumptions $1-4$ be fulfilled with $\sqrt{2}\left(\gamma_{1}+\gamma_{2}+\gamma_{3}\right)<\frac{c_{3}}{c_{4}}$. Then in the closed loop system the equilibrium point $\left(x_{1}^{*}, x_{2}^{*}, z^{*}\right)$ is locally asymptotically stable, the control objective (2) is achieved, and the matched uncertainty is compensated completely.

Proof: In order to show stability of the closed loop dynamics, it is sufficient to show stability of the reduced dynamics as the choice of $z(0)=-g\left(x_{1}(0), x_{2}(0)\right)$ and condition (15) ensures the initialisation and existence of the sliding mode for all times. We write the reduced dynamics (8) in relative coordinates $\Delta x_{1}, \Delta x_{2}$ and $\Delta z$, that is

$$
\begin{aligned}
& \Delta \dot{x}_{1}=f_{1}\left(x_{1}^{*}+\Delta x_{1}, l\left(x_{1}^{*}+\Delta x_{1}, z^{*}+\Delta z\right)\right) \\
& +\phi_{1}\left(x_{1}^{*}+\Delta x_{1}, l\left(x_{1}^{*}+\Delta x_{1}, z^{*}+\Delta z\right)\right), \\
& \Delta \dot{z}=h\left(x_{1}^{*}+\Delta x_{1}, l\left(x_{1}^{*}+\Delta x_{1}, z^{*}+\Delta z\right)\right) .
\end{aligned}
$$

Defining the perturbation $\zeta=\left[\begin{array}{ll}\zeta_{1}^{\top} & \zeta_{2}\end{array}\right]$ with

$$
\begin{aligned}
\zeta_{1}\left(\Delta x_{1}, \Delta z\right):= & f_{1}\left(x_{1}, l\left(x_{1}, z\right)\right)-f_{1}\left(\Delta x_{1}, l\left(\Delta x_{1}, \Delta z\right)\right) \\
& +\phi_{1}\left(x_{1}, l\left(x_{1}, z\right)\right) \\
\zeta_{2}\left(\Delta x_{1}, \Delta z\right):= & h\left(x_{1}, l\left(x_{1}, z\right)\right)-h\left(\Delta x_{1}, l\left(\Delta x_{1}, \Delta z\right)\right) .
\end{aligned}
$$

we obtain the perturbed system

$$
\begin{aligned}
\Delta \dot{x}_{1} & =f_{1}\left(\Delta x_{1}, l\left(\Delta x_{1}, \Delta z\right)\right)+\zeta_{1}\left(\Delta x_{1}, \Delta z\right), \\
\Delta \dot{z} & =h\left(\Delta x_{1}, l\left(\Delta x_{1}, \Delta z\right)\right)+\zeta_{2}\left(\Delta x_{1}, \Delta z\right) .
\end{aligned}
$$

Substituting (10), (11) and (14) yields

$$
\begin{aligned}
\left\|\zeta_{1}\left(\Delta x_{1}, \Delta z\right)\right\|= & \| f_{1}\left(x_{1}, l\left(x_{1}, z\right)\right)-f_{1}\left(\Delta x_{1}, l\left(\Delta x_{1}, \Delta z\right)\right) \\
& -f_{1}\left(x_{1}^{*}, l\left(x_{1}^{*}, z^{*}\right)\right)-\phi_{1}\left(x_{1}^{*}, l\left(x_{1}^{*}, z^{*}\right)\right) \\
& +\phi_{1}\left(x_{1}, l\left(x_{1}, z\right)\right) \| \\
\leq & \| f_{1}\left(x_{1}, l\left(x_{1}, z\right)\right)-f_{1}\left(\Delta x_{1}, l\left(\Delta x_{1}, \Delta z\right)\right) \\
& -f_{1}\left(x_{1}^{*}, l\left(x_{1}^{*}, z^{*}\right)\right) \| \\
& +\left\|\phi_{1}\left(x_{1}, l\left(x_{1}, z\right)\right)-\phi_{1}\left(x_{1}^{*}, l\left(x_{1}^{*}, z^{*}\right)\right)\right\| \\
\leq & \left(\gamma_{1}+\gamma_{2}\right)\|\Delta w\|, \\
\left\|\zeta_{2}\left(\Delta x_{1}, \Delta z\right)\right\|= & \left\|h\left(x_{1}, l\left(x_{1}, z\right)\right)-h\left(\Delta x_{1}, l\left(\Delta x_{1}, \Delta z\right)\right)\right\| \\
\leq & \gamma_{3}\|\Delta w\| .
\end{aligned}
$$

Using the Euclidean norm and Hölder's inequality we obtain

$$
\begin{aligned}
\left\|\zeta\left(\Delta x_{1}, \Delta z\right)\right\| & \leq \sqrt{2}\left(\left\|\zeta_{1}\left(\Delta x_{1}, \Delta z\right)\right\|+\left\|\zeta_{2}\left(\Delta x_{1}, \Delta z\right)\right\|\right) \\
& \leq \sqrt{2}\left(\gamma_{1}+\gamma_{2}+\gamma_{3}\right)\|\Delta w\|
\end{aligned}
$$

Consider the Lyapunov function $V$ of Assumption 3 for system (16b) and take its time derivative

$$
\begin{aligned}
\dot{V}(\Delta w) & =\frac{\partial V}{\partial \Delta w}\left(\left[\begin{array}{c}
f_{1}\left(\Delta x_{1}, l\left(\Delta x_{1}, \Delta z\right)\right) \\
h\left(\Delta x_{1}, l\left(\Delta x_{1}, \Delta z\right)\right)
\end{array}\right]+\zeta(\Delta w)\right) \\
& \leq \frac{\partial V}{\partial \Delta w}\left[\begin{array}{c}
f_{1}\left(\Delta x_{1}, l\left(\Delta x_{1}, \Delta z\right)\right) \\
h\left(\Delta x_{1}, l\left(\Delta x_{1}, \Delta z\right)\right)
\end{array}\right]+\left\|\frac{\partial V}{\partial \Delta w} \zeta(\Delta w)\right\| .
\end{aligned}
$$

With (12) and (17) we obtain

$$
\dot{V}(\Delta w) \leq-c_{3}\|\Delta w\|^{2}+c_{4} \sqrt{2}\left(\gamma_{1}+\gamma_{2}+\gamma_{3}\right)\|\Delta w\|^{2} .
$$

Thus $\dot{V}(\Delta w)$ is strictly negative if $\sqrt{2}\left(\gamma_{1}+\gamma_{2}+\gamma_{3}\right)<\frac{c_{3}}{c_{4}}$.

This renders the equilibrium point $\left(x_{1}^{*}, z^{*}\right)$ of system $(8)$ asymptotically stable which implies that $\lim _{t \rightarrow \infty} z$ exist. Hence, in view of (3) the time integral of $h$ takes a limit. Since $h$ is uniformly continuous, with Barbălat's Lemma we conclude that $\lim _{t \rightarrow \infty} h\left(x_{1}, x_{2}\right)=0$. Furthermore, due to the initialisation (7) the closed-loop system is in slidingmode for $t \geq 0$ and thus the matched uncertainty $\phi_{2}$ is compensated completely.

Remark 3 In [6] similar arguments are used as in Theorem 2 to show stability of a conventional integral sliding mode controller.

\section{B. Comparison to conventional integral sliding mode}

In conventional integral sliding mode as proposed in [1] with several design propositions, e.g. [7],[19],[8], a sliding manifold and integral state is designed based on a conventionally designed nominal control $u_{0}$ where

$$
\begin{aligned}
\dot{z} & =-G(x) f(x)-G_{2}(x) b_{2}(x) u_{0}(x), \\
s\left(x_{1}, x_{2}, z\right) & =g\left(x_{1}, x_{2}\right)+z .
\end{aligned}
$$

Then, the reduced dynamics are the same as in the nominal case but without the influence of the matched uncertainty. Unmatched uncertainties can be compensated to the extent of the nominal control $u_{0}$.

If we cast our approach into this scheme then we can identify the continuous nominal control $u_{0}$ and the discontinuous control $u_{1}$ such that $u=u_{0}+u_{1}$ in terms of

$$
\begin{aligned}
& u_{0}(x)=\frac{-1}{G_{2}(x) b_{2}(x)}(G(x) f(x)+h(x)), \\
& u_{1}(x)=\frac{-1}{G_{2}(x) b_{2}(x)} \rho \operatorname{sgn}(s) .
\end{aligned}
$$

An overview of the two different design methods for an integral sliding mode controller is given by Table I.

In the conventional design all effort has to be put into the choice of the nominal control $u_{0}$ to achieve the control objective (2). In particular, the integrator state is determined by the choice of $u_{0}$ and is not chosen, as in our proposal, directly to guarantee the control objective. Furthermore, the choice of $g$ in the switching function does not influence the reduced dynamics in $\left(x_{1}, x_{2}\right)$. In the proposed method $g$ influences the reduced dynamics significantly and thus allows to shape the desired dynamics.

Furthermore, the order of the reduced dynamics in the conventional design is $n$ plus the order needed for the nominal 
controller. Our approach results in reduced dynamics of order $n$. Thus, assuming a nominal control with integral action for the conventional design, our proposed approach will always yield reduced dynamics of lower order. This may lead to a simpler stability analysis and less restrictive requirements.

Note also, since our proposed method can be cast into the standard framework, results obtained for integral sliding mode also extend to our method. For example, the choice of $G(x)^{\top}=\left[\begin{array}{ll}0 & b_{2}(x)\end{array}\right]$ minimises the effect of an unmatched disturbance in the sense of [8].

\section{Decoupling of unmatched disturbances}

In this section, we consider a special case of (1) and show that the switching function can be designed such that at least some components of the unmatched disturbance are fully compensated for all $t \geq 0$ if $h\left(x_{1}, x_{2}\right)=h^{\mathrm{d}}\left(x_{11}\right)$ depending only on some component of $x_{1}$. Let $x_{1}(t)$ be split into $x_{11}(t) \in \mathbb{R}^{n-2}$ and $x_{12}(t) \in \mathbb{R}$ such that system (1) reads

$$
\begin{aligned}
\dot{x}_{11} & =f_{11}\left(x_{11}, x_{12}, x_{2}\right)+\phi_{11}\left(x_{11}\right) \\
\dot{x}_{12} & =f_{12}\left(x_{11}, x_{12}, x_{2}\right)+\phi_{12}\left(x_{11}, x_{12}, x_{2}\right) \\
\dot{x}_{2} & =f_{2}\left(x_{1}, x_{2}\right)+b_{2}\left(x_{1}, x_{2}\right) u+\phi_{2}\left(t, x_{1}, x_{2}\right) .
\end{aligned}
$$

Theorem 4 Consider system (20) augmented by (3) with the integral sliding mode control law (6) and initialisation (7). Let Assumptions 1-4 be fulfilled with $\sqrt{2}\left(\gamma_{1}+\gamma_{2}+\gamma_{3}\right)<\frac{c_{3}}{c_{4}}$.

Choose the integrator state (3) such that

$$
\dot{z}=h^{\mathrm{d}}\left(x_{11}\right)
$$

and the switching function (4) such that the first component of the reduced dynamics (8) is independent of $x_{12}$, i.e.

$$
f_{11}^{\mathrm{d}}\left(x_{11}, z\right):=f_{11}\left(x_{11}, x_{12}, l\left(x_{11}, x_{12}, z\right)\right) .
$$

Then in the closed loop system the equilibrium point $\left(x_{1}^{*}, x_{2}^{*}, z^{*}\right)$ is locally asymptotically stable, the control objective (2) is fulfilled, the matched uncertainty $\phi_{2}$, and the unmatched uncertainty $\phi_{12}$ are compensated completely on $h$ for all $t \geq 0$.

Proof: The asymptotic stability directly follows from Theorem 2. For the reduced dynamics (8) with (20) and (22) we have

$$
\begin{aligned}
\dot{x}_{11}= & f_{11}^{\mathrm{d}}\left(x_{11}, z\right)+\phi_{11}\left(x_{11}\right) \\
\dot{x}_{12}= & f_{12}\left(x_{11}, x_{12}, l\left(x_{11}, x_{12}, z\right)\right) \\
& +\phi_{12}\left(x_{11}, x_{12}, l\left(x_{11}, x_{12}, z\right)\right) \\
\dot{z}= & h^{\mathrm{d}}\left(x_{11}\right) .
\end{aligned}
$$

From the reduced dynamics of $x_{11}$ and $z$ in (23a) and (23c) we can see that the disturbance $\phi_{12}$ does not influence the dynamics of $x_{11}$, neither directly nor via the influence of states disturbed by $\phi_{12}$.

Remark 5 If (23) is bounded-input bounded-state stable for input $\phi_{12}$ and state $x_{12}$ then we can also allow that $\phi_{12}$ shows an additional time-varying component, e.g. an external disturbance input, without violating control objective (2).

Remark 6 The decoupling conditions (21), (22) can also be achieved using other (non-integral) sliding-mode techniques as shown in the numerical example in Section $V$.

\section{SPecial CASE: Linear System}

In this section we shall consider the linear case and apply our proposed ISM design. Consider system (1) with

$$
\begin{aligned}
\dot{x}_{11} & =A_{11} x_{11}+A_{12} x_{12}+A_{13} x_{2}+\phi_{11}\left(x_{11}\right) \\
\dot{x}_{12} & =A_{21} x_{11}+A_{22} x_{12}+A_{23} x_{2}+\phi_{12}\left(x_{11}, x_{12}, x_{2}\right) \\
\dot{x}_{2} & =A_{31} x_{11}+A_{32} x_{12}+A_{33} x_{2}+u+\phi_{2}\left(t, x_{11}, x_{12}, x_{2}\right)
\end{aligned}
$$

where $x_{11}(t) \in \mathbb{R}^{n-2}$ and $x_{12}(t), x_{2}(t), u(t) \in \mathbb{R}$. Note that this type of system results generically from a time-invariant nonlinear system where only some linear part is certain and the nonlinearities are considered as uncertainties $\phi_{i}$.

The output of interest in view of (2) is

$$
y=h(x)=\left[\begin{array}{lll}
H_{11} & H_{12} & H_{2}
\end{array}\right] x .
$$

\section{A. Proposed ISM with disturbance decoupling}

We choose the integral state $z$ to comply with

$$
\dot{z}=h\left(x_{1}, x_{2}\right)=H_{11} x_{11}+H_{12} x_{12}+H_{2} x_{2}
$$

and select the switching function

$$
s=G_{11} x_{11}+G_{12} x_{12}+G_{2} x_{2}+z
$$

with $G_{11} \in \mathbb{R}^{n-2}$ and $G_{12}, G_{2} \in \mathbb{R}$ such that the sliding manifold $s \equiv 0$ is given by

$$
x_{2}=l\left(x_{11}, x_{12}, z\right)=-\frac{G_{11}}{G_{2}} x_{11}-\frac{G_{12}}{G_{2}} x_{12}-\frac{1}{G_{2}} z .
$$

We obtain the reduced dynamics

$$
\left[\begin{array}{c}
\dot{x}_{11} \\
\dot{x}_{12} \\
\dot{z}
\end{array}\right]=A^{\text {red }}\left[\begin{array}{c}
x_{11} \\
x_{12} \\
z
\end{array}\right]+\left[\begin{array}{c}
\phi_{11}\left(x_{1}\right) \\
\phi_{12}\left(x_{11}, x_{12}, l\left(x_{11}, x_{12}, z\right)\right) \\
0
\end{array}\right],
$$

with

$$
A^{\mathrm{red}}=\left[\begin{array}{ccc}
A_{11}-A_{13} \frac{G_{11}}{G_{2}} & A_{12}-A_{13} \frac{G_{12}}{G_{2}} & -A_{13} \frac{1}{G_{2}} \\
A_{21}-A_{23} \frac{G_{11}}{G_{2}} & A_{22}-A_{23} \frac{G_{12}}{G_{2}} & -A_{23} \frac{1}{G_{2}} \\
H_{11}-H_{2} \frac{G_{11}}{G_{2}} & H_{12}-H_{2} \frac{G_{12}}{G_{2}} & -H_{2} \frac{1}{G_{2}}
\end{array}\right] .
$$

In view of conditions (21) and (22) of Theorem 4 we may decouple the uncertainty $\phi_{12}$ from the desired output $h$ if

$$
A_{13} \frac{G_{12}}{G_{2}}=A_{12}, \quad H_{12}=0, \quad H_{2}=0 .
$$

The remaining parameters $G_{11}, G_{2}$ can be used to shape the dynamics of the desired output $H x$. Substituting (28) into (27), we are left to design the state feedback

$$
\left[\begin{array}{c}
\dot{x}_{11} \\
\dot{z}
\end{array}\right]=\left[\begin{array}{ll}
A_{11} & 0 \\
H_{11} & 0
\end{array}\right]\left[\begin{array}{c}
x_{11} \\
z
\end{array}\right]-\left[\begin{array}{c}
A_{13} \\
0
\end{array}\right] K\left[\begin{array}{c}
x_{11} \\
z
\end{array}\right]
$$




\begin{tabular}{|c|c|c|}
\hline & Conventional ISMC design method & Proposed ISMC design method \\
\hline Design parameter & Nominal Control $u_{0}$ & Dynamic of the integrator state $h$ \\
\hline Switching function & $\begin{array}{l}\text { Switching function via } g \\
s\left(x_{1}, x_{2}, z\right)=g\left(x_{1}, x_{2}\right)+z \\
s\left(x_{1}, x_{2}, z\right)=0 \Leftrightarrow x_{2}=l\left(x_{1}, z\right)\end{array}$ & $\begin{array}{l}\text { Switching function via } g \\
s\left(x_{1}, x_{2}, z\right)=g\left(x_{1}, x_{2}\right)+z \\
s\left(x_{1}, x_{2}, z\right)=0 \Leftrightarrow x_{2}=l\left(x_{1}, z\right)\end{array}$ \\
\hline Control law & $u=u_{0}+u_{1}$ & $\begin{array}{l}u=u_{0}+u_{1} \\
u_{0}=-\left[G_{2}(x) b_{2}(x)\right]^{-1}(G(x) f(x)+h(x))\end{array}$ \\
\hline & $u_{1}=-\left[G_{2}(x) b_{2}(x)\right]^{-1} \rho \operatorname{sgn}(s)$ & $u_{1}=-\left[G_{2}(x) b_{2}(x)\right]^{-1} \rho \operatorname{sgn}(s)$ \\
\hline Integrator state & $\dot{z}=-G(x) f(x)-G_{2}(x) b_{2}(x) u_{0}(x)$ & $\dot{z}=h\left(x_{1}, x_{2}\right)$ \\
\hline Red. dyn. in $\left(x_{1}, x_{2}\right)$ & $\begin{array}{l}\dot{x}_{1}=f_{1}(x)+\phi_{1}(x) \\
\dot{x}_{2}=f_{2}(x)+b_{2}(x) u_{0}(x)-b_{2}(x) G_{1}(x) \phi_{1}(x)\end{array}$ & $\begin{array}{l}\dot{x}_{1}=f_{1}(x)+\phi_{1}(x) \\
\dot{x}_{2}=-\left[G_{2}(x)\right]^{-1}\left(G_{1}(x) f_{1}(x)-h(x)\right)-b_{2}(x) G_{1}(x) \phi_{1}(x)\end{array}$ \\
\hline $\begin{array}{l}\text { Red. dyn. in }\left(x_{1}, z\right) \\
\left(w=\left(x_{1}, l\left(x_{1}, z\right)\right)\right)\end{array}$ & $\begin{array}{l}\dot{x}_{1}=f_{1}\left(x_{1}, l\left(x_{1}, z\right)\right)+\phi_{1}\left(x_{1}, l\left(x_{1}, z\right)\right. \\
\dot{z}=-G(w) f(w)-G_{2}(w) b_{2}(w) u_{0}(w)\end{array}$ & $\begin{array}{l}\dot{x}_{1}=f_{1}\left(x_{1}, l\left(x_{1}, z\right)\right)+\phi_{1}\left(x_{1}, l\left(x_{1}, z\right)\right) \\
\dot{z}=h\left(x_{1}, l\left(x_{1}, z\right)\right)\end{array}$ \\
\hline Condition on $\rho$ & $\rho>\left\|G_{1} \phi_{1}+G_{2} \phi_{2}\right\|$ & $\rho>\left\|G_{1} \phi_{1}+G_{2} \phi_{2}\right\|$ \\
\hline
\end{tabular}

TABLE I

COMPARISON OF ISMC DESIGN METHODS FOR SYSTEM (1).

with $K=\left[\begin{array}{ll}\frac{G_{11}}{G_{2}} & \frac{1}{G_{2}}\end{array}\right]$. Additionally, stability of $x_{12}$ shall be guaranteed in the nominal case $\phi_{1} \equiv 0$, which results in

$$
A_{22}-A_{23} \frac{G_{12}}{G_{2}}<0 .
$$

Thus, the design (29) is to meet the constraints (28) and (30).

It remains to check Assumptions 1-4 of Theorem 2. Assumption 1 requires a stationary solution. This solution is trivial if the perturbation is vanishing at the origin, i.e. $\phi(0)=0$. Assumption 2 is fulfilled since we deal with a linear system. Assumption 3 is fulfilled by the choice of $K$ in (29) and condition (30). Assumption 4 needs to be checked with some knowledge about the uncertainty. We may then choose $\rho>\phi^{\text {sup }}$ as in Theorem 1 to ensure the existence of the sliding mode.

\section{Simulation Example}

In this section we illustrate the proposed design with an example and compare the results to conventional ISM design, [7], [19], [8]. In the spirit of the example in [19] we consider the following system with $x=\left[\begin{array}{lll}x_{11} & x_{12} & x_{2}\end{array}\right]^{\top} \in \mathbb{R}^{3}$ :

$$
\begin{aligned}
\dot{x}_{11} & =x_{12}+x_{2}+\phi_{11}\left(x_{11}\right) \\
\dot{x}_{12} & =x_{2}+\phi_{12}(t, x) \\
\dot{x}_{2} & =f_{2}(t, x)+b_{2}(t, x) u+\phi_{2}(t, x)
\end{aligned}
$$

with

$$
\begin{aligned}
& f_{2}(t, x)=x_{11} x_{12}-x_{2} \sin \left(x_{11}\right)+\cos (t) \\
& b_{2}(t, x)=3+\frac{2}{\pi} \arctan \left(x_{2}\right)+0.5 \sin (t) .
\end{aligned}
$$

Note that we inherit the time-varying uncertainty $\phi_{12}$ from the example in [19]. Such uncertainty is not covered by Theorem 4. However, the example shows that the approach is appropriate for time-varying uncertainty in some cases.

The control objective is to obtain an asymptotically stable equilibrium and $\lim _{t \rightarrow \infty} h(x)=0$ with desired output $h(x)=x_{11}$. We use our proposed method to design an ISMC which achieves the control objective and also decouples two of three process states from the uncertainty. For comparison, we take two conventionally designed ISMCs. For the nominal control we consider a PI state-feedback that achieves the control objective as well as a sliding mode control law satisfying (21) for decoupling. The sliding manifold is chosen according to [7] such that the discontinuous part does not amplify the effect of the unmatched uncertainties $\phi_{11}, \phi_{12}$.

\section{A. Proposed ISM design}

According to (3) and (7), we choose:

$$
\dot{z}=H x=\left[\begin{array}{lll}
1 & 0 & 0
\end{array}\right] x, \quad z(0)=-G x(0)
$$

and the switching function shall be parametrised as in (25) with sliding-manifold (26). From the decoupling condition (28), we get the requirement $\frac{G_{12}}{G_{2}}=1$. This also satisfies the stability requirement (30) for the internal state $x_{12}$.

For the remaining dynamics we consider (29)

$$
\left[\begin{array}{c}
\dot{x}_{11} \\
\dot{z}
\end{array}\right]=\left[\begin{array}{ll}
0 & 0 \\
1 & 0
\end{array}\right]\left[\begin{array}{c}
x_{11} \\
z
\end{array}\right]-\left[\begin{array}{l}
1 \\
0
\end{array}\right] K\left[\begin{array}{c}
x_{11} \\
z
\end{array}\right] .
$$

Choosing the eigenvalues $\lambda_{1,2}=-1$ we obtain $G_{11}=2$ and $G_{2}=1$ which also determines $G_{12}=1$.

Applying the control law (6) we require $\rho>\phi^{\text {sup }} \geq\|G \phi\|$.

\section{B. Conventional ISMC design}

For comparison we design two nominal controls. Each of them is augmented by a sliding-mode control to compensate the matched uncertainties.

1) Nominal PI state-feedback controller: A PI statefeedback control law is suitable to compensate stationary uncertainties, choosing the integrator state $v$ with $\dot{v}=x_{11}$. The nominal control law is then given by

$$
u_{0}(x)=\frac{1}{b_{2}(t, x)}\left(K\left(\begin{array}{l}
x \\
v
\end{array}\right)-f_{2}(t, x)\right)
$$

with $K \in \mathbb{R}^{1 \times 4}$. The state-feedback matrix $K$ is to be designed for the closed loop system

$$
\left[\begin{array}{c}
\dot{x}_{11} \\
\dot{x}_{12} \\
\dot{x}_{2} \\
\dot{v}
\end{array}\right]=\left[\begin{array}{cccc}
0 & 1 & 1 & 0 \\
0 & 0 & 1 & 0 \\
0 & 0 & 0 & 0 \\
1 & 0 & 0 & 0
\end{array}\right]\left[\begin{array}{c}
x_{11} \\
x_{12} \\
x_{2} \\
v
\end{array}\right]+\left[\begin{array}{c}
0 \\
0 \\
1 \\
0
\end{array}\right] K\left[\begin{array}{c}
x_{11} \\
x_{12} \\
x_{2} \\
v
\end{array}\right] .
$$




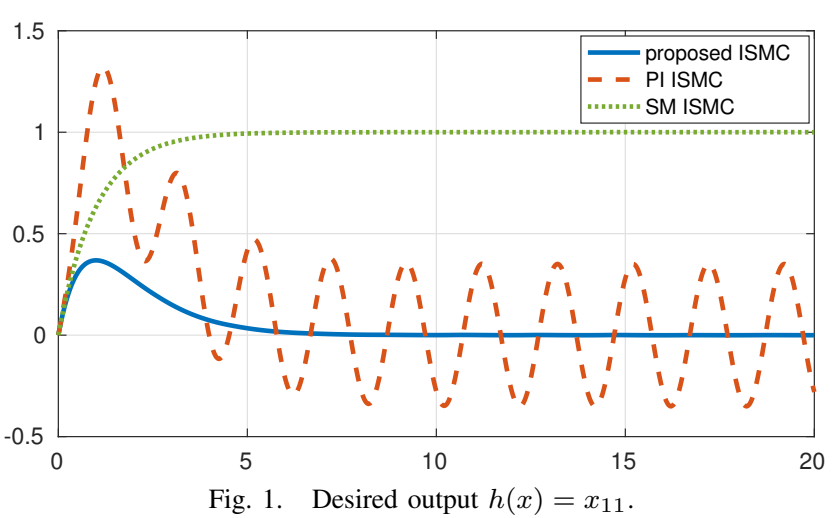

Choosing all four eigenvalues as -1 yields

$$
K=\left[\begin{array}{llll}
-3 & -3 & -4 & -1
\end{array}\right] .
$$

2) Nominal sliding-mode control: The decoupling condition (28) may also be used to design a switching function $s^{\mathrm{SM}}=G^{\mathrm{SM}} x$ with $G^{\mathrm{SM}}:=\left[\begin{array}{lll}G_{11}^{\mathrm{SM}} & G_{12}^{\mathrm{SM}} & G_{2}^{\mathrm{SM}}\end{array}\right]$ for a nominal sliding-mode controller.

For the reduced dynamics $s^{\mathrm{SM}} \equiv 0$ we obtain:

$$
\left[\begin{array}{l}
\dot{x}_{11} \\
\dot{x}_{12}
\end{array}\right]=\left[\begin{array}{cc}
-\frac{G_{11}^{\mathrm{SM}}}{G_{2}^{\mathrm{SM}}} & 1-\frac{G_{12}^{\mathrm{SM}}}{G_{2}^{\mathrm{SM}}} \\
1 & 0
\end{array}\right]\left[\begin{array}{l}
x_{11} \\
x_{12}
\end{array}\right] .
$$

Thus, decoupling is obtained for $G_{12}^{\mathrm{SM}}=G_{2}^{\mathrm{SM}}$. Choosing the remaining eigenvalue as -1 , we get $G^{\mathrm{SM}}=\left[\begin{array}{lll}1 & 1 & 1\end{array}\right]$. With $\rho^{\mathrm{SM}}>\left\|G^{\mathrm{SM}} \phi\right\|$ the sliding-mode control reads

$$
u_{0}(x)=\frac{-1}{G^{\mathrm{SM}} b(t, x)}\left(G^{\mathrm{SM}} f(t, x)+\rho^{\mathrm{SM}} \operatorname{sgn}\left(s^{\mathrm{SM}}\right)\right),
$$

where $b(t, x)=\left[\begin{array}{lll}0 & 0 & b_{2}(t, x)\end{array}\right]^{\top}$.

3) ISMC law: For compensating the matched uncertainty $\phi_{2}$ we pick the sliding manifold as $s^{\mathrm{ISMC}}=x_{2}+z$ with integrator state $z$ as in (18). The complete ISM control is

$$
u=u_{0}-\rho^{\mathrm{ISMC}} \operatorname{sgn}\left(s^{\mathrm{ISMC}}\right),
$$

with $\rho^{\mathrm{ISMC}}>\left\|\phi_{2}\right\|$ and nominal control $u_{0}$ in (32) or (33).

\section{Simulation Results}

We simulate the system (31) with the disturbances

$$
\begin{aligned}
\phi_{11}\left(x_{11}\right) & =1, \\
\phi_{12}\left(t, x_{11}, x_{12}, x_{2}\right) & =4 \sin (\pi t), \\
\phi_{2}\left(t, x_{11}, x_{12}, x_{2}\right) & =\left\{\begin{array}{l}
2 \text { for } 5(2 k) \leq t<5(2 k+1) \\
0 \text { for } 5(2 k+1) \leq t<5(2(k+1))
\end{array}\right.
\end{aligned}
$$

with $k \in \mathbb{N}$. The systems are initialised at the origin and the sliding-mode gain is chosen as $\rho^{\mathrm{SM}}=\rho=10$ to dominate the uncertainty in each case. In order to avoid chattering we approximate $\operatorname{sgn}(s) \approx \frac{s}{|s|+\varepsilon}$ with $\varepsilon=0.001$.

Note, that the reduced dynamics of the PI-ISCM is of fourth order, whereas the SM-ISCM and our proposed ISCM have reduced dynamics of third order.

Fig. 1 shows the evolution of the desired output $h(x)=$ $x_{11}$. Under the proposed control law, the matched disturbance

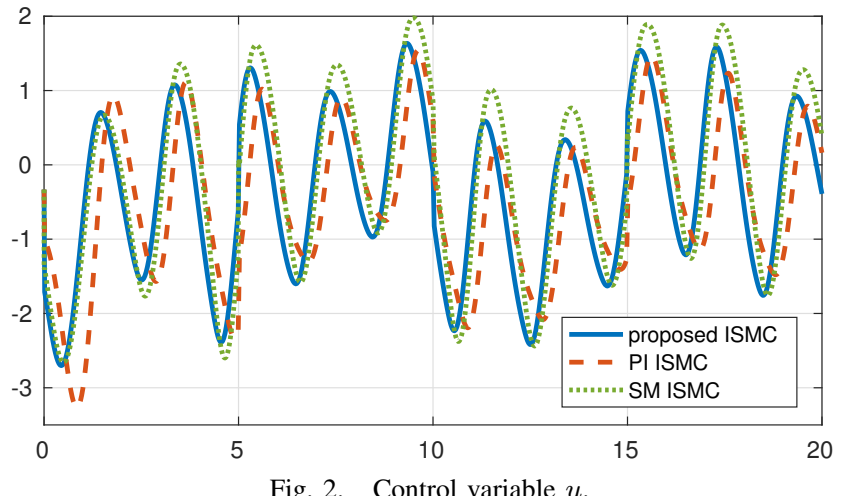

Fig. 2. Control variable $u$.

$\phi_{2}$ as well as the unmatched disturbance $\phi_{12}$ do not influence the course of the state at all. The unmatched uncertainty $\phi_{11}$ influences the transient behaviour of the state but is completely compensated by the integrator for $t \rightarrow \infty$. The PI-ISMC compensates the matched disturbance $\phi_{2}$ and the stationary influence of $\phi_{11}$, but the unmatched disturbance $\phi_{12}$ shows a strong impact. The ISMC with SM nominal control compensates the matched uncertainty $\phi_{2}$ also decouples the unmatched uncertainty $\phi_{12}$, but the unmatched uncertainty $\phi_{11}$ causes a stationary error.

Fig. 2 shows the control variable of the three control laws. The matched uncertainty $\phi_{2}$ is compensated by all three control laws which causes the discontinuity of the control variables at 5,10 and 15 time units. The unmatched uncertainty $\phi_{11}$ results in an offset of the control variable of the SM-ISMC compared to the other two control laws.

\section{CONCLUSION}

We have presented a novel and systematic design method for an integral sliding mode controller for nonlinear systems in regular form. The approach is capable of compensating matched and also a class of unmatched uncertainties. Moreover, we derive conditions that allow for complete decoupling of a class of unmatched uncertainties. The design is focused on the direct choice of output for which stationary accuracy is required. The resulting controller can be cast into the standard framework of integral sliding mode approaches and a direct comparison of the approaches is provided. Our proposed approach integrates the nominal control and the sliding manifold into one design procedure. Whenever integral action is included in the nominal control of the conventional design approach our proposed design result in dynamics of lower order. For linear systems, the stability and decoupling conditions enable a very simple design procedure that is readily applicable. A simulation example illustrates the design and highlights the compensation and decoupling properties in comparison to conventional ISMC.

\section{ACKNOWLEDGMENT}

The authors kindly acknowledge support by the European Union Horizon 2020 research and innovation program under Marie Skłodowska-Curie grant agreement No. 734832. 


\section{REFERENCES}

[1] V. Utkin and J. Shi, "Integral sliding mode in systems operating under uncertainty conditions," in Conference on Decision and Control, 1996, pp. 4591-4596.

[2] J. H. Kim, S. A. Gadsden, and S. A. Wilkerson, "Adaptive integral sliding mode controller for longitudinal rotation control of a tilt-rotor aircraft," in Mediterranean Conference on Control and Automation, 2016, pp. 820-825.

[3] J.-X. Xu, Z.-Q. Guo, and T. H. Lee, "Design and implementation of integral sliding-mode control on an underactuated two-wheeled mobile robot," IEEE Transactions on Industrial Electronics, vol. 61, no. 7, pp. 3671-3681, 2014.

[4] J. Yang, S. Li, J. Su, and X. Yu, "Continuous nonsingular terminal sliding mode control for systems with mismatched disturbances," Automatica, vol. 49, no. 7, pp. 2287-2291, 2013.

[5] J. Davila, "Exact tracking using backstepping control design and high-order sliding modes," IEEE Transactions on Automatic Control, vol. 58, no. 8, pp. 2077-2081, 2013.

[6] W.-J. Cao and J.-X. Xu, "Nonlinear integral-type sliding surface for both matched and unmatched uncertain systems," IEEE Transactions on Automatic Control, vol. 49, no. 8, pp. 1355-1360, 2004.

[7] F. Castaños and L. Fridman, "Analysis and design of integral sliding manifolds for systems with unmatched perturbations," IEEE Transactions on Automatic Control, vol. 51, no. 5, pp. 853-858, 2006.

[8] M. Rubagotti, A. Estrada, F. Castaños, A. Ferrara, and L. Fridman, "Integral sliding mode control for nonlinear systems with matched and unmatched perturbations," IEEE Transactions on Automatic Control, vol. 56, no. 11, pp. 2699-2704, 2011.

[9] A. Estrada and L. Fridman, "Integral HOSM semiglobal controller for finite-time exact compensation of unmatched perturbations," IEEE Transactions on Automatic Control, vol. 55, no. 11, pp. 2645-2649, 2010.

[10] —, "Exact compensation of unmatched perturbation via quasicontinuous HOSM," in Conference on Decision and Control, 2008, pp. 2202-2207.

[11] A. Estrada, L. Fridman, and R. Iriarte, "Combined backstepping and HOSM control design for a class of nonlinear MIMO systems,"
International Journal of Robust and Nonlinear Control, vol. 27, no. 4, pp. 566-581, 2016.

[12] Y. Bin, K. Li, and N. Feng, "Disturbance decoupling robust control of vehicle full speed cruise dynamic system," Science in China Series E: Technological Sciences, vol. 52, no. 12, pp. 3545-3564, 2009.

[13] S. C. Thomsen and N. K. Poulsen, "A disturbance decoupling nonlinear control law for variable speed wind turbines," in Mediterranean Conference on Control \& Automation, 2007, pp. 1-6.

[14] A. Ferreira de Loza, E. Puntab, L. Fridman, G. Bartolini, and S. Delprat, "Nested backward compensation of unmatched perturbations via HOSM observation," Journal of the Franklin Institute, vol. 351, no. 5, pp. 2397-2410, 2014.

[15] A. Ferreira de Loza, J. Cieslak, D. Henry, A. Zolghadri, and L. Fridman, "Output tracking of systems subjected to perturbations and a class of actuator faults based on HOSM observation and identification," Automatica, vol. 59, pp. 200-205, 2015.

[16] T. S. Gabbi, H. A. Gründling, and R. P. Vieira, "Sliding mode current control based on disturbance observer applied to permanent magnet synchronous motor," in Brazilian Power Electronics Conference and Southern Power Electronics Conference, 2015, pp. 122-127.

[17] A. Louk'yanov and V. Utkin, "Methods of reducing equations of dynamic systems to regular form," Automation and Remote Control, vol. 42, no. 4, pp. 413-420, 1981.

[18] Y. Shtessel, C. Edwards, L. Fridman, and A. Levant, Sliding mode control and observation. Birkhäuser, 2013.

[19] M. Rubagotti, A. Estrada, F. Castaños, A. Ferrara, and L. Fridman, "Optimal disturbance rejection via integral sliding mode control for uncertain systems in regular form," in International Workshop on Variable Structure Systems, 2010, pp. 78-82. 